\title{
Optimal Flows On Road Networks In Emergency Cases
}

\author{
Nicola Pasquino \\ Department of Industrial Engineering \\ University of Salerno \\ Via Ponte Don Melillo, 84084, Fisciano (SA), Italy \\ Email: nipasquino@unisa.it
}

\author{
Luigi Rarità \\ Centre of Research for Pure and Applied Mathematics \\ c/o Department of Electronic and Computer Engineering \\ University of Salerno \\ Via Ponte Don Melillo, 84084, Fisciano (SA), Italy \\ Email:1rarita@unisa.it
}

\begin{abstract}
In this paper, we present a technique to redistribute car flows, described by a fluid dynamic model, on a portion of the Caltanissetta city, in Italy, when critical situations, such as car accidents, occur. Using a decentralized approach, a cost functional, that describes the asymptotic average velocity of emergency vehicles, is maximized with respect to distribution coefficients at simple junctions with two incoming roads and two outgoing ones. Then, in order to manage critical situations in high traffic conditions, local optimal coefficients at each node of the network are used. The overall traffic evolution is studied via simulations, that confirm the goodness of the optimization results. It is also proved that optimal coefficients allow a fast transit of emergency vehicles on assigned routes on the network.
\end{abstract}

Keywords-conservation laws; traffic problems; redistribution of flows

\section{Introduction}

Road networks are always characterized by a high car density and congestions, leading to queues formations, difficulties in forecasting travel times, pollution problems, etc. Heavy traffic levels often produce car accidents, with consequent problems for the emergency management. In such a context, some techniques for managing road traffic in emergency cases represent a topic of great importance. The aim of this paper is to use some optimization results on a portion of Caltanissetta urban network, Italy, in order to redistribute traffic flows in such a way that emergency vehicles can travel at the maximum allowed speed along assigned roads.

A fluid dynamic model is used: the evolution of car densities is described on each road by a conservation law ([4], [7], [8]), while dynamics at junctions of $n \times m$ type ( $n$ incoming roads and $m$ outgoing ones) is uniquely solved using rules for the traffic distributions at nodes and right of ways (if $n>m$ ). Considering the distribution coefficients as control parameters, we propose to redirect traffic at $2 \times 2$ junctions in order to face emergency situations. In particular, assuming that emergency vehicles will cross assigned roads ([6]), it is considered a cost functional $W_{(\varphi, \psi)}$, measuring, for $2 \times 2$ junctions, the average velocities of such vehicles on the incoming road $I_{\varphi}, \varphi \in\{1,2\}$, and the outgoing road $I_{\psi}$, $\psi \in\{3,4\}$. The optimization results give the values of the distribution coefficients, which maximize the functional, allowing a fast transit of emergency vehicles to reach car accident's place and hospital.

As the analysis of $W_{(\varphi, \psi)}$ on a whole network is very complex, a decentralized approach is considered, namely: the asymptotic behaviour (for large times) is assumed and an exact solution of $W_{(\varphi, \psi)}$ is found at a single $2 \times 2$ junction.

Then, we propose a global (sub)optimal solution for the whole network, simply obtained applying at each junction of $2 \times 2$ type the computed local optimal solution. A similar decentralized procedure has been studied for different types of road junctions and various functionals in [2], [3], and [5].

The optimization results are tested by simulations. Two choices of distribution coefficients are evaluated: optimal values given by the optimization algorithm, and random values, i.e. at the beginning of the simulation process, random values of traffic parameters are kept constant during all simulations. For the case study of a portion of the Caltanissetta urban network in Italy, it is proved that the choice of optimal distribution coefficients at $2 \times 2$ junctions allows better performances on the network. Moreover, on the basis of an algorithm described in [1] for tracing car trajectories on networks, some simulations are run to test how the distribution coefficients influence the total travelling time of emergency vehicles. It is shown that the time for covering a path of a single emergency vehicle decreases when optimal parameters are considered.

The paper is organized as follows. In Section II, the model for car traffic is introduced. Section III deals with the definition of the cost functional for emergency vehicles and the optimization of traffic coefficients. Simulations for the case study are presented in Section IV. The paper ends with conclusions in Section V.

\section{A Model for Car Traffic on Networks}

A road network is described by a couple $(\bar{I}, \bar{J})$, where $\bar{I}$ is the set of roads, modelled by intervals $\left[a_{i}, b_{i}\right] \subset R$,

Identify applicable sponsor/s here. (sponsors) 
$i=1, \ldots, N$, and $\bar{J}$ is the collection of junctions. Indicating by $\rho=\rho(t, x) \in\left[0, \rho_{\max }\right]$ the density of cars, $\rho_{\max }$ the maximal density, $f(\rho)=\rho v(\rho)$ the flux with $v(\rho)$ the average velocity, the traffic dynamics is described on each road by the conservation law (Lighthill-Whitham-Richards model, [7], [8]):

$$
\partial_{t} \rho+\partial_{x} f(\rho)=0
$$

We assume that: (F) $f$ is a strictly concave $C^{2}$ function such that $f(0)=f\left(\rho_{\max }\right)=0$. Considering a decreasing velocity function:

$$
v(\rho)=v_{\max }\left(1-\rho / \rho_{\max }\right), \rho \in\left[0, \rho_{\max }\right]
$$

and setting $v_{\max }=\rho_{\max }=1$, a flux function fulfilling (F) is:

$$
f(\rho)=\rho(1-\rho), \rho \in[0,1],
$$

which has a unique maximum $\sigma=1 / 2$. Dynamics at junctions is described solving Riemann Problems (RPs), Cauchy Problems with a constant initial datum for each incoming and outgoing road.

Fix a junction $J$ of $n \times m$ type ( $n$ incoming roads $I_{\varphi}$, $\varphi=1, \ldots, n$, and $m$ outgoing roads, $\left.I_{\psi}, \psi=n+1, \ldots, n+m\right)$ and an initial datum $\rho_{0}=\left(\rho_{1,0}, \ldots, \rho_{n, 0}, \rho_{n+1,0}, \ldots, \rho_{n+m, 0}\right)$. A Riemann Solver (RS) for the junction $J$ is a map $R S:[0,1]^{n} \times[0,1]^{m} \rightarrow[0,1]^{n} \times[0,1]^{m}$ that associates to $\rho_{0}$ a vector 際 $\left(\rho_{1}, \ldots\right.$, 袋, $\left., \rho_{n+1}, \ldots, \rho_{n+m}\right)$ so that the solution on an incoming road $I_{\varphi}, \varphi=1, \ldots, n$, is the wave $\left(\rho_{\varphi, 0}, \hat{\rho}_{\varphi}\right)$ and on an outgoing one $I_{\psi}, \psi=n+1, \ldots, n+m$ is the wave $\left(\hat{\rho}_{\psi}, \rho_{\psi, 0}\right)$. We require the following conditions hold true: (C1) $R S\left(R S\left(\rho_{0}\right)\right)=R S\left(\rho_{0}\right)$; (C2) on each incoming road, the wave $\left(\rho_{\varphi, 0}, \hat{\rho}_{\varphi}\right)$ has negative speed, while, on each outgoing road, the wave $\left(\hat{\rho}_{\psi}, \rho_{\psi, 0}\right)$ has positive speed.

If $m \geq n$, a possible RS at $J$ is defined by the following rules (see [4]): (A) traffic is distributed at $J$ distribution matrix $A=\left(\alpha_{\psi, \varphi}\right), \quad \varphi=1, \ldots, n$, $\psi=n+1, \ldots, n+m, \quad 0<\alpha_{\psi, \varphi}<1, \quad \sum_{j=n+1}^{n+m} \alpha_{\psi, \varphi}=1$. The $\varphi$-th column of $A$ indicates the percentages of traffic that, from the incoming road $I_{\varphi}$, distribute to the outgoing roads; (B) respecting (A), drivers maximize the flux through $J$. according to some coefficients, collected in a traffic

If $n>m$, a further rule (a yielding criterion) is necessary: (C) Assume that not all cars can enter the outgoing roads, and let $Q$ be the amount that can do it. Then, $p_{\varphi} Q$ cars come from the incoming road $I_{\varphi}$, where $\left.p_{\varphi} \in\right] 0,1\left[\right.$ is the right of way parameter of $\operatorname{road} I_{\varphi}$, $\varphi=1, \ldots, n$, and $\sum_{\varphi=1}^{n} p_{\varphi}=1$.

Focus on a junction $J$ of $2 \times 2$ type (incoming roads $I_{1}$ and $I_{2}$, and outgoing roads $I_{3}$ and $I_{4}$ ). We indicate the cars density on incoming and outgoing roads, respectively, by $\rho_{\varphi}(t, x) \in[0,1], \quad(t, x) \in R^{+} \times I_{\varphi} \quad, \quad \varphi=1,2 \quad$, $\rho_{\psi}(t, x) \in[0,1],(t, x) \in R^{+} \times I_{\psi}, \psi=3,4$. From condition (C2), fixing the flux function and assuming $\rho_{0}=\left(\rho_{1,0}, \rho_{2,0}, \rho_{3,0}, \rho_{4,0}\right)$ as the initial datum of an RP at $J$, the maximal flux values on roads are defined by:

$$
\begin{aligned}
& \gamma_{\varphi}^{\max }=\left\{\begin{array}{ll}
f\left(\rho_{\varphi, 0}\right), & \text { if } 0 \leq \rho_{\varphi, 0} \leq 1 / 2, \\
f(1 / 2), & \text { if } 1 / 2 \leq \rho_{\varphi, 0} \leq 1,
\end{array} \varphi=1,2,\right. \\
& \gamma_{\psi}^{\max }=\left\{\begin{array}{ll}
f(1 / 2), & \text { if } 0 \leq \rho_{\psi, 0} \leq 1 / 2, \\
f\left(\rho_{\psi, 0}\right), & \text { if } 1 / 2 \leq \rho_{\psi, 0} \leq 1,
\end{array} \quad \psi=3,4\right.
\end{aligned}
$$

In this case, the traffic distribution matrix $A$ has the coefficients $\alpha_{3,1}, \alpha_{3,2}, \alpha_{4,1}=1-\alpha_{3,1}, \alpha_{4,2}=1-\alpha_{3,2}$, and the assumption $\alpha_{3,1} \neq \alpha_{3,2}$ is made to guarantee the uniqueness of solutions. From rules (A) and (B), it follows that the flux solution to the RP at $J$, 涳 $\left(\gamma_{1}\right.$, 疼, $\left.\gamma_{3}, \gamma_{4}\right)$, is found as follows: the incoming fluxes $\hat{\gamma}_{\varphi}, \varphi=1,2$, are solutions of the problem $\max \left(\gamma_{1}+\gamma_{2}\right)$, with $0 \leq \gamma_{\varphi} \leq \gamma_{\varphi}^{\max }$, $0 \leq \alpha_{\psi, 1} \gamma_{1}+\alpha_{\psi, 2} \gamma_{2} \leq \gamma_{\psi}^{\max }, \varphi=1,2, \psi=3,4$. The outgoing fluxes $\hat{\gamma}_{\psi}, \psi=3,4, \quad$ are simply given by 淘 $=\alpha_{\psi, 1} \gamma_{1}+\alpha_{\psi, 2} \gamma_{2}$. Once $\hat{\gamma}$ is known, $\hat{\rho}$ is found as follows:

$$
\hat{\rho}_{\varphi} \in\left\{\begin{array}{ll}
\left.\left.\left\{\rho_{\varphi, 0}\right\} \cup\right] \tau\left(\rho_{\varphi, 0}\right), 1\right], & \text { if } 0 \leq \rho_{\varphi, 0} \leq 1 / 2, \\
{[1 / 2,1],} & \text { if } 1 / 2 \leq \rho_{\varphi, 0} \leq 1
\end{array} \varphi=1,2\right.
$$

$\hat{\rho}_{\varphi} \in\left\{\begin{array}{ll}{[0,1 / 2],} & \text { if } 0 \leq \rho_{\psi, 0} \leq 1 / 2, \\ \left.\left.\left\{\rho_{\psi, 0}\right\} \cup\right] \tau\left(\rho_{\psi, 0}\right), 1\right], & \text { if } 1 / 2 \leq \rho_{\psi, 0} \leq 1,\end{array} \psi=3,4\right.$, 
where $\tau:[0,1] \rightarrow[0,1]$ is the map such that $f(\tau(\rho))=f(\rho) \forall \rho \in[0,1]$, $\tau(\rho) \neq \rho \forall \rho \in[0,1] \backslash\{1 / 2\}$.

\section{Optimization of Traffic Coefficients}

Assume that a car accident occurs on a road of an urban network and that some emergency vehicles have to the reach the place of the accident, or a hospital. We define the velocity function for such vehicles as:

$$
\omega(\rho)=1-\delta+\delta v(\rho)
$$

with $0<\delta<1$ and $v(\rho)$ as in. Since $\omega\left(\rho_{\max }\right)=1-\delta>0$, it follows that the emergency vehicles travel with a higher velocity with respect to cars. For a junction $J$ of $2 \times 2$ type (incoming roads $I_{1}$ and $I_{2}$; outgoing roads $I_{3}$ and $I_{4}$ ), given the initial datum $\left(\rho_{1,0}, \rho_{2,0}, \rho_{3,0}, \rho_{4,0}\right)$, the cost functional $W_{(\varphi, \psi)}(t)$, which indicates the average velocity of emergency vehicles crossing the incoming road $I_{\varphi}$, $\varphi \in\{1,2\}$, and the outgoing road $I_{\psi}, \psi \in\{3,4\}$, is defined as:

$$
W_{(\varphi, \psi)}(t):=\int_{I_{\varphi}} \omega\left(\rho_{\varphi}(t, x)\right) d x+\int_{I_{\psi}} \omega\left(\rho_{\psi}(t, x)\right) d x
$$

For the case $\varphi=1$ and $\psi=3$, we have the following theorem, proved in [6] (for other combinations of $\varphi$ and $\psi$, the statement is similar).

Theorem III-1. Consider a junction $J$, with incoming roads $I_{1}$ and $I_{2}$, and outgoing roads $I_{3}$ and $I_{4}$. For $t=T>>$, the parameters $\alpha_{3,1}$ and $\alpha_{3,2}$, which maximize the cost functional $W_{(1,3)}(t)$, are $\alpha_{3,1}^{\text {opt }}=1-\frac{\gamma_{4}^{\max }}{\gamma_{1}^{\max }}$, $0 \leq \alpha_{3,2}^{\text {opt }}<1-\frac{\gamma_{4}^{\max }}{\gamma_{1}^{\max }}$, with the exception of the following cases, where the optimal values do not exist and are approximated: for $\varepsilon_{1}$ and $\varepsilon_{2}$ small, positive and such that $\varepsilon_{1} \neq \varepsilon_{2}$, if $\gamma_{1}^{\max } \leq \gamma_{4}^{\max }, \alpha_{3,1}^{\text {opt }}=\varepsilon_{1}, \alpha_{3,2}^{\text {opt }}=\varepsilon_{2}$; otherwise, if $\gamma_{1}^{\max }>\gamma_{3}^{\max }+\gamma_{4}^{\max }$, then $\quad \alpha_{3,1}^{\text {opt }}=\frac{\gamma_{3}^{\max }}{\gamma_{3}^{\max }+\gamma_{4}^{\max }}-\varepsilon_{1} \quad$ and $\alpha_{3,2}^{o p t}=\frac{\gamma_{3}^{\max }}{\gamma_{3}^{\max }+\gamma_{4}^{\max }}-\varepsilon_{2}$

\section{Simulations}

The validity of the optimization results stated in Theorem III-1 is studied considering different control procedures, applied locally at each junction, on the global behaviour of a real network. Such analysis is completed by computing the travelling time of an emergency vehicle on assigned paths.

We focus on a portion of the urban network of Caltanissetta, Italy, see Fig. 1. The network consists of: 8 roads, identified by 51 segments (see Table I), whose eight ones are incoming roads $(1,5,23,27,35,39,46,51)$, and nine ones are outgoing roads $(2,4,8,22,25,34,37,44,49)$; 25 nodes of different types: $2 \times 2$, identified as $A_{i}$, $i=1, \ldots, 11 ; 2 \times 1$, labelled by $B_{i}, i=1, \ldots, 6 ; 1 \times 2$, indicated by $C_{i}, i=1, \ldots, 7 ; 1 \times 1, D_{1}$.

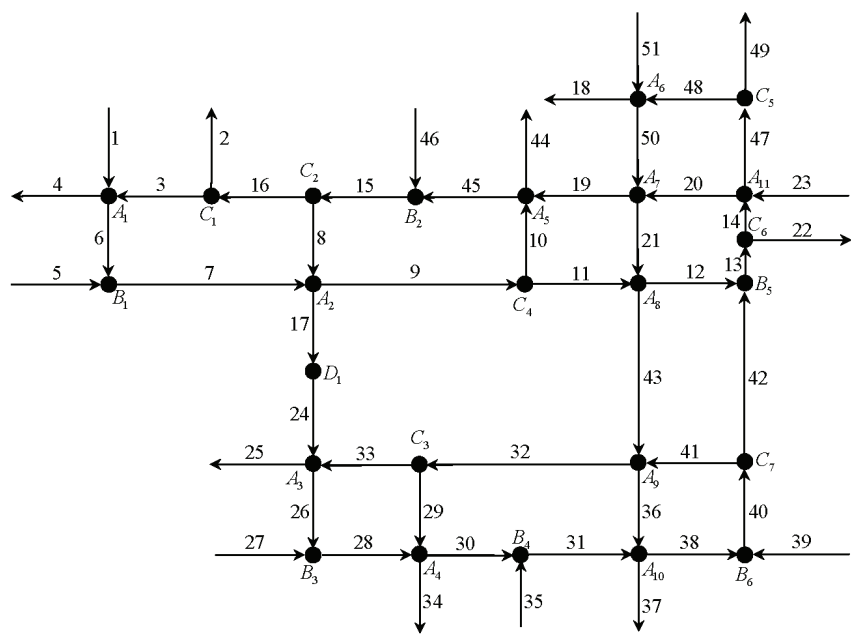

Fig. 1. Topology of a portion of Caltanissetta network

TABLE I CORRESPONDENCE AMONG ROADS AND NUMBERS IN FIG. 1

\begin{tabular}{|c|c|}
\hline Real roads & Graph road/segments \\
\hline Via Giuseppe Mulè & 1,2 \\
\hline Via Luigi Monaco & $3-21$ \\
\hline Via della Regione & 22,23 \\
\hline Via Due Fontane & $24-33$ \\
\hline Via SD1 & 34,35 \\
\hline Via Leone XIII & $36-43$ \\
\hline Via Luigi Russo & $44,45,46$ \\
\hline Via Poggio S. Elia & $47-51$ \\
\hline
\end{tabular}

We assume that emergency vehicles follow the path $P=\Lambda_{1} \cup \Lambda_{2} \cup \Lambda_{3} \cup \Lambda_{4}$ with $\Lambda_{1}=\{23,47,48,50,19,45,15\}, \Lambda_{2}=\{16,3,6,7,17,24,26\}$, $\Lambda_{3}=\{28,30,31,38,40,42,13\} \quad$ and $\Lambda_{4}=\{14,20,21,43,32,33,35\}$. Hence, we analyze the behaviour of the cost functional $J(t)=\sum_{(\varphi, \psi) \in \Gamma} W_{(\varphi, \psi)}(t)$, with $W_{(\varphi, \psi)}(t)$ defined as in and $\Gamma:=\left\{\begin{array}{c}(23,47),(48,50),(50,19),(19,45),(3,6),(7,17), \\ (24,26),(28,30),(31,38),(20,21),(43,32),(33,25)\end{array}\right\}$.

Traffic flows simulations are made using the Godunov method with $\Delta x=0.01, \Delta t=\Delta x / 2$ in a time interval $[0, T]$, with $T=150 \mathrm{~min}$. Initial conditions and boundary 
data for densities have been chosen approaching $\rho_{\max }=1$, with the aim of simulating a congestion scenario on the network, and are the following: initial datum equal to 0.8 for all roads; boundary data 0.9 for roads $1,5,23,27$ and 35; 0.95 for roads 39,46 , and $51 ; 0.8$ for roads $2,4,18,22$ and $25 ; 0.85$ for roads $34,37,44$ and 49 . According to measures on the real network, we set, for junctions $B_{i}, i=1, \ldots, 6$, the following right of way parameters: $p_{12}=p_{26}=0.2$, $p_{46}=0.3, p_{6}=p_{35}=0.4, p_{38}=p_{39}=0.5, p_{5}=p_{30}=0.6$, $p_{45}=0.7, p_{42}=p_{27}=0.8$; for junctions $C_{i}, i=1, \ldots, 7$, the following distribution coefficients: $\alpha_{49,47}=\alpha_{22,13}=0.3$, $\alpha_{8,15}=\alpha_{33,32}=0.4 \quad, \quad \alpha_{41,40}=0.2$ $\alpha_{2,16}=\alpha_{3,16}=\alpha_{10,9}=\alpha_{11,9}=0 \quad, \quad \alpha_{42,40}=0.8 \quad$, $\alpha_{16,15}=\alpha_{29,32}=0.6 \quad, \quad \alpha_{48.47}=\alpha_{14,13}=0.7 \quad$. Moreover, $\delta=0.5$ is considered.

We analyze two different simulation cases: locally optimal distribution coefficients (optimal case) at each junction $A_{i}, i=1, \ldots, 11$, i. e. parameters according to Theorem III-1; random parameters (random case), namely the distribution coefficients are taken randomly for each junction $A_{i}, i=1, \ldots, 11$, when the simulation starts and then are kept constant.

In Fig. 2, the behaviour of the cost functional $J(t)$ is represented. The optimal simulation is indicated by a continuous line, while random cases by dashed curves. As expected, random simulations lines of $J(t)$ are always lower than the optimal one. In fact, when optimal parameters are used, junctions of $2 \times 2$ type are interested by a congestion reduction, due to the flows redistribution on roads. Even if right of way parameters of junctions $B_{i}$, $i=1, \ldots, 6$, and distribution coefficients of junctions $C_{i}$, $i=1, \ldots, 7$, are optimized using the results in [2] and [3], traffic conditions are almost unaffected.

Suppose that an emergency vehicle travels along a path in a network. Its position $x=x(t)$ is obtained solving the Cauchy problem:

$$
\left\{\begin{array}{l}
\dot{x}=\omega(\rho(t, x)), \\
x\left(t_{0}\right)=x_{0},
\end{array}\right.
$$

where $x_{0}$ is the initial position at the initial time $t_{0}$. Using numerical methods, described in [1], it is possible to estimate the travelling time of the emergency vehicle. First, we compute the trajectory along road 45 and the time needed for covering it in optimal case and random cases; then, we consider the path $P$ and study the exit time evolution versus the initial travel time $t_{0}$ (the time in which the emergency vehicle enters into the network).

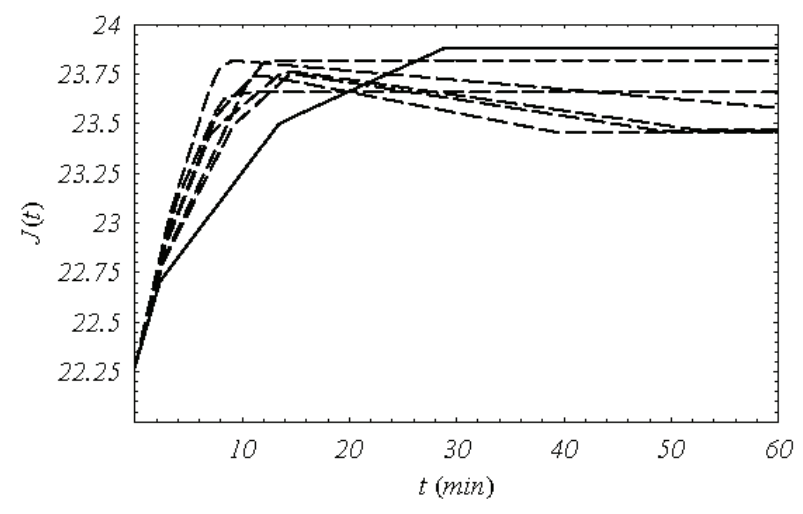

Fig. 2. Evolution of $J(t)$ in $[0,60]$ using optimal distribution coefficients (continuous line) and random choices (dashed lines)

In Fig. 3, we assume that the emergency vehicle starts its own travel at the beginning of road 45 at the initial time $t_{0}=40$ and compute the trajectories $x(t)$ along road 45, in optimal (continuous line) and random cases (dashed lines).

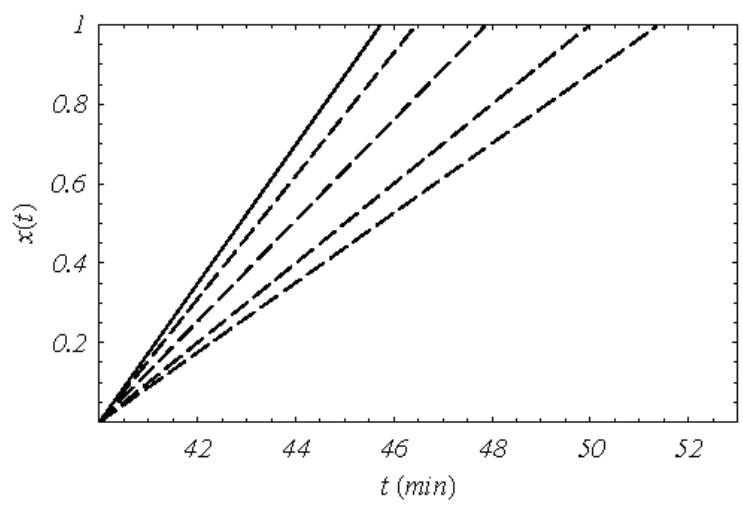

Fig. 3. Trajectory $x(t)$ for an emergency vehicle along road 45 with $t_{0}=40$, optimal coefficients (continuous line) and random choices (dashed lines)

The evolution $x(t)$ in the optimal case has always a higher slope with respect to trajectories in random cases because traffic levels are low. When random distribution coefficients are used, shocks propagating backwards increase the density values on the whole network; the velocity for the emergency vehicles is reduced and exit times from road 45 become longer. In Table II, assuming $t_{0}=40$ we report the time instants $t_{\text {out }}$ in which the emergency vehicle goes out of road 45, either for the optimal values of distribution coefficients (opt) or random choices $\left(r_{i}, i=1, . ., 4\right)$.

TABLE II. TIMES $t_{\text {out }}$, ASSUMING $t_{0}=40$
\begin{tabular}{|c|c|c|c|c|c|}
\hline Simulations & opt & $r_{1}$ & $r_{2}$ & $r_{3}$ & $r_{4}$ \\
\hline$t_{\text {out }}$ & 45.70 & 51.34 & 49.94 & 47.84 & 46.42 \\
\hline
\end{tabular}


The exit time $T_{\text {exit }}\left(t_{0}\right)=t_{\text {out }}-t_{0}$ from road 45 versus the initial time $t_{0}$, assuming that the emergency vehicle starts its path from the beginning of the road, is depicted in Fig. 4. Notice that the choice of optimal coefficients (continuous line) allows to obtain an exit time lower than the other cases (dashed lines), due to decongestion effects. The exit time becomes stable after a certain initial time value $\left(t_{0}=9.45\right.$ for the optimal distribution choice, unlike the random cases, for which $t_{0}=5.1, t_{0}=5.3, t_{0}=6.3$ and $t_{0}=7.4$ ).

In Fig. 5, we report the exit time $T_{\text {exit }}\left(t_{0}\right)$ from the chosen path $P$ versus the initial times $t_{0}$. When optimal parameters are not used, $T_{\text {exit }}\left(t_{0}\right)$ is the lowest curve, as expected, because the network is not congested. Furthermore, $T_{\text {exit }}\left(t_{0}\right)$ never tends to infinity as the emergency vehicle has a higher velocity with respect to cars, hence it is able to reach its own destination although some roads of the chosen path are completely blocked. The steady value of $T_{\text {exit }}\left(t_{0}\right)$ is reached at time $t_{0}=6.9$.

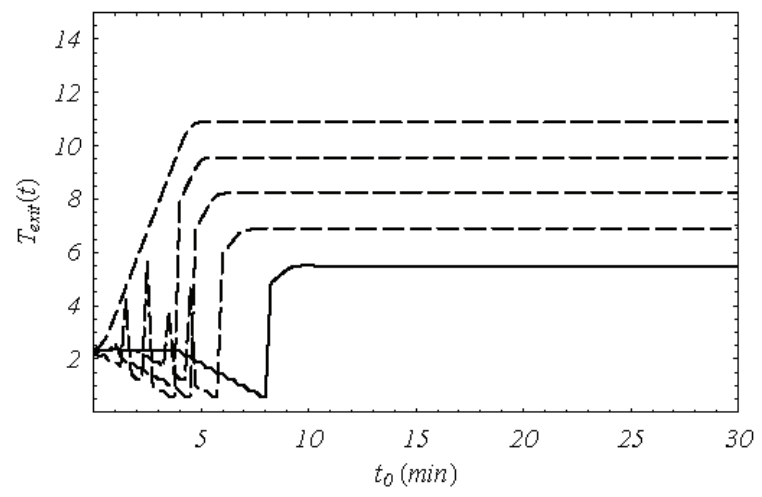

Fig. 4. Exit time from road 45 vs $t_{0}$ in $[0,30]$; optimal coefficients (continuous line) and random choices (dashed lines).

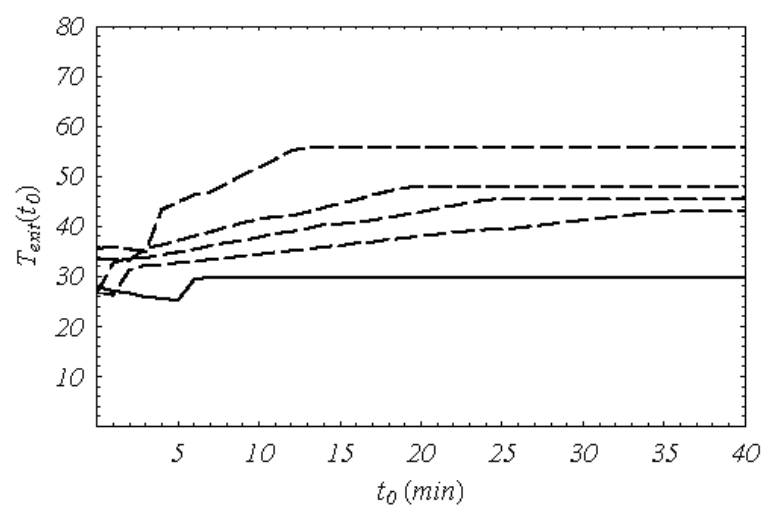

Fig. 5. Exit time from the path $\mathrm{P}$ vs $t_{0}$ in $[0,40]$; optimal coefficients (continuous line) and random choices (dashed lines)

\section{Conclusions}

In this paper, it is presented an optimization study to manage emergency situations on road networks. Optimal distribution coefficients at road junctions with two incoming roads and two outgoing ones have been computed maximizing a cost functional, that measures the average velocity of emergency vehicles. Simulations have been made on a real urban network. It has been proved, through a numerical evaluation of emergency vehicles trajectories, that fast transits are possible also in cases of high congestions.

\section{REFERENCES}

[1] G. Bretti, and B. Piccoli, "A tracking algorithm for car paths on road networks," SIAM Journal on Applied Dynamical Systems, vol. 7, pp. 510-531, 2008.

[2] A. Cascone, C. D'Apice, B. Piccoli, and L. Rarità, "Optimization of traffic on road networks," Mathematical Models and Methods in Applied Sciences, vol. 17, no. 10, pp. 1587-1617, 2007.

[3] A. Cascone, C. D'Apice, B. Piccoli, and L. Rarità, "Circulation of car traffic in congested urban areas," Communication in Mathematical Sciences, vol. 6, no. 3, pp. 765-784, 2008.

[4] G. Coclite, M. Garavello, and B. Piccoli, "Traffic Flow on Road Networks," SIAM Journal on Mathematical Analysis, vol. 36, no. 6, pp. 1862-1886, 2005.

[5] C. D'Apice, R. Manzo, and L. Rarità, "Splitting of Traffic Flows to Control Congestion in Special Events," International Journal of Mathematics and Mathematical Sciences, vol. 2011, pp. 1-18, 2011, Article ID 563171, doi:10.1155/2011/563171.

[6] R. Manzo, B. Piccoli, and L. Rarità, "Optimal distribution of traffic flows at junctions in emergency cases," European Journal of Applied Analysis, vol. 23, no. 4, pp. 515-535, 2012.

[7] M. J. Lighthill, and G. B. Whitham, "On kinetic waves. II. Theory of Traffic Flows on Long Crowded Roads," Proc. Roy. Soc. London Ser. A, vol. 229, pp. 317-345, 1955.

[8] P. I. Richards, "Shock Waves on the Highway," Oper. Res., vol. 4, pp. 42-51, 1956. 